\title{
Clinical biomarkers directing the management of patients with colon and lung cancer (beyond on- cogene-addicted NSCLC]
}

Ioannis Ntanasis-Stathopoulos ${ }^{1}$, Anastasios Kyriazoglou', Meletios A. Dimopoulos ${ }^{1}$, Maria Gavriatopoulou ${ }^{1}$

${ }^{1}$ Department of Clinical Therapeutics, National and Kapodistrian University of Athens, School of Medicine, Alexandra General Hospital, Athens, Greece

Received 26 August 2019; Accepted 5 November 2019

\begin{abstract}
Treatment personalisation plays a key role in the current management of patients with cancer. Several biomarkers have shown clinical utility and may guide therapeutic decisions. Amongst patients with lung cancer, the level of expression of programmed death ligand 1 (PD-L1) has both prognostic and predictive values in terms of response to the inhibition of programmed cell death protein 1 (PD-1). Depending on the clinical setting, the expression of $\mathrm{PD}-\mathrm{L} 1 \geq 1 \%$ or $\geq 50 \%$ has been associated with improved outcomes amongst patients receiving pembrolizumab. Regarding patients with colorectal carcinoma, mutations in the KRAS oncogene predict the responsiveness to the inhibition of epidermal growth factor receptor (EGFR). Only patients with wild-type KRAS tumours derive benefit from cetuximab and panitumumab in terms of response and survival. In conclusion, future research should aim in the optimisation of the use of biomarker in the clinical practice in order to provide the optimal drug combination to each individual patient.
\end{abstract}

Keywords: biomarker • therapeutics $\bullet$ cancer $\bullet P D-L 1 \cdot K R A S$

\section{Rapid communication}

The significant progress in drug development regarding the field of oncology during the past decades has resulted in the availability of a large number of anticancer therapies. However, the heterogeneity in individual tumour characteristics and host variables results in differential effects of each drug to each tumour type and each unique patient. Therefore, there is a need for a tailored therapeutic approach, as reviewed in [1]. In this context, biological markers have emerged as key indicators of treatment outcomes and have been already incorporated in therapeutic algorithms. A biomarker is defined as an objective indicator of normal or pathogenic processes and may have predictive and prognostic value [2]. Herein, the biomarker-guided management of patients with lung and colon cancer is presented critically.

Lung cancer constitutes the most frequently diagnosed malignancy and the leading cause of cancerattributed deaths worldwide [3]. Although targeted agents have significantly improved the outcomes of patients with lung adenocarcinoma and epidermal growth factor receptor (EGFR), anaplastic lymphoma kinase (ALK) or ROS proto-oncogene 1 mutations, as reviewed in [4], these highly effective treatments are applicable only in a subset of patients. Immune checkpoint blockade has emerged as a novel strategy for activating the host immune response against cancer cells, especially regarding non-small-cell lung cancer (NSCLC), as reviewed in [5]. Pembrolizumab is a programmed cell 
death protein 1 (PD-1) inhibitor that was evaluated in the cardinal phase $2 / 3 \mathrm{KEYNOTE-010} \mathrm{clinical} \mathrm{trial}$ [6]. A total of 1,034 patients with NSCLC stage IIIB/IV who had received at least one prior line of therapy and presented with at least $1 \%$ expression of programmed death ligand 1 (PD-L1) were randomly assigned (1:1:1) to receive either pembrolizumab at a dose of 2 or $10 \mathrm{mg} /$ $\mathrm{kg}$ or docetaxel at a dose of $75 \mathrm{mg} / \mathrm{m}^{2}$ every 3 weeks. Pembrolizumab at a dose of either 2 or $10 \mathrm{mg} / \mathrm{kg}$ was superior to docetaxel in terms of overall survival (OS) (hazard ratio $[\mathrm{HR}]=0.71,95 \%$ confidence interval $[\mathrm{Cl}]$ : $0.58-0.88 ; p=0.0008$ and $\mathrm{HR}=0.61,95 \% \mathrm{Cl}$ : 0.49 0.75 ; $p<0.0001$, respectively). Regarding progressionfree survival (PFS), pembrolizumab was non-inferior to docetaxel. Interestingly, the efficacy of pembrolizumab was more pronounced in the subset of patients with the expression of PD-L1 on at least $50 \%$ of tumour cells. Both OS and PFS were significantly prolonged with pembrolizumab at a dose of $2 \mathrm{mg} / \mathrm{kg}$ versus docetaxel (14.9 months vs. 8.2 months [median]; $\mathrm{HR}=0.54$, 95\% Cl: $0.38-0.77 ; p=0.0002$ and 5.0 months vs. 4.1 months; $\mathrm{HR}=0.59,95 \% \mathrm{Cl}: 0.44-0.78 ; \mathrm{p}=0.0001$, for OS and PFS, respectively) and pembrolizumab at a dose of $10 \mathrm{mg} / \mathrm{kg}$ compared with docetaxel (17.3 months vs. 8.2 months; $\mathrm{HR}=0.50,95 \% \mathrm{Cl}$ : $0.36-$ $0.70 ; \mathrm{p}<0.0001$ and 5.2 months vs. 4.1 months; $\mathrm{HR}=$ 0.59, 95\% Cl: 0.45-0.78; p<0.0001, for OS and PFS respectively). In a recently updated analysis with a longer median follow-up of 31 months, pembrolizumab retained its survival benefit over docetaxel regardless of the use of archival or fresh samples for the assessment of PD-L1 [7]. Pembrolizumab has also been evaluated as an upfront treatment in the phase 3 KEYNOTE-024 study that included 305 newly diagnosed patients with advanced NSCLC and at least $50 \%$ of expression of PDL1 in the absence of EGFR or ALK mutations and were randomised $(1: 1)$ to receive either pembrolizumab at the fixed dose of $200 \mathrm{mg}$ every 3 weeks or platinum-based chemotherapy [8]. The median PFS was prolonged with pembrolizumab compared with chemotherapy (10.3 months v. 6 months, $\mathrm{HR}=0.50,95 \% \mathrm{Cl}: 0.37-0.68$; $p<0.001$ ), whereas in the recently published, updated analysis of the median OS was also higher amongst patients receiving pembrolizumab compared with those receiving chemotherapy (30 months vs. 14.2 months, $\mathrm{HR}=0.63,95 \% \mathrm{Cl}: 0.47-0.86)$ [8, 9]. KEYNOTE-189 included a similar patient population with KEYNOTE-024 in addition to patients with no or $<50 \%$ of expression of PD-L1 [10]. About 616 patients were randomised (2:1) to receive pemetrexed and platinum-based treatment with or without pembrolizumab. The superiority of the pembrolizumab group was more pronounced amongst patients with $\geq 1 \%$ of expression of PD-L1 compared with those with $<1 \%$ of expression of PD-L1 both in terms of PFS (HR [95\% Cl]: 0.44 [0.34-0.57] vs. 0.75 [0.53-1.05], respectively) and OS (HR, [95\% Cl]: 0.47 (0.34-0.66) vs. 0.59 [0.38-0.92], respectively). Recently, a $\geq 1 \%$ expression of PD-L1 was also associated with a higher overall response rate (ORR) compared with $<1 \%$ expression ( $41 \%$ vs. $15 \%$, respectively) amongst patients with advanced NSCLC receiving the first-line combination treatment with the PD-1 inhibitor nivolumab and the cytotoxic T-lymphocyte-associated antigen 4 (CTLA-4) inhibitor ipilimumab [11]. Therefore, the expression of PD-L1 drives therapeutic decisions in the contemporary management of patients with lung cancer.

Colorectal carcinoma significantly contributes to the global cancer burden regarding both incidence and mortality [3]. Therefore, a personalised approach is deemed necessary in order to optimise patient outcomes. EGFR molecular cascade plays a key role in carcinogenesis in colon; its aberrant activation promotes both cell proliferation and survival, as reviewed in [12]. Anti-EGFR monoclonal antibodies, namely, cetuximab and panitumumab, have shown significant efficacy and have been incorporated in the therapeutic algorithm for patients with colon cancer [13]. However, not all patients derive benefit from this treatment approach. KRAS is an intracellular GTPase that is activated following the dimerisation and autophosphorylation of the transmembrane EGFR receptor and is the main intracellular signal transducer [12]. Mutations in KRAS gene lead to a permanently activated KRAS protein irrespective of EGFR status. Thus, the inhibition of EGFR does not alter the status of KRAS protein, and KRAS mutations have a predictive value in terms of the response to anti-EGFR monoclonal antibodies. In a series of 59 patients with metastatic colorectal carcinoma who were refractory to chemotherapy and were subsequently treated with the combination of chemotherapy and cetuximab, the presence of KRAS mutations was significantly associated with disease progression ( $p=0.0005$ ) and decreased the time to progression ( $p=0.015)$ [14]. Furthermore, a larger series, including data from 89 patients, showed that KRAS mutations were associated with resistance to cetuximab $(p<0.001)$, shorter PFS (median: 10.1 weeks vs. 31.4 weeks; $p=0.0001$ ) and OS (median: 10.1 months vs. 14.3 months; $p=0.026$ ) [15]. Another study with a larger number of patients $(n=394)$ with advanced colorectal cancer that had not responded to chemotherapy showed that cetuximab with best supportive care was superior to best supportive care alone amongst patients with wildtype KRAS tumours in terms of both PFS (median: 3.7 months vs. 1.9 months; $p<0.001$ ) and OS (median: 9.5 months vs. 4.8 months; $p<0.001$ ) [16]. In a similar study 
setting comparing panitumumab monotherapy $(n=208)$ with best supportive care $(n=219)$, the absence of KRAS mutations was a positive predictor for response and longer PFS [17]. Importantly, a pooled analysis of the large, randomised CRYSTAL and OPUS clinical trials including 845 patients confirmed the benefit of addition of cetuximab in the first-line treatment of wildtype KRAS metastatic colorectal cancer regarding ORR $(p<0.0001)$, PFS $(p<0.001)$ and OS $(p=0.0062)$ [18]. The addition of panitumumab in the upfront treatment of patients with metastatic colorectal carcinoma significantly improved PFS (median: 9.6 months vs. 8.0 months; $p=0.02$ ) in the phase 3 PRIME study, as well [19]. Interestingly, a specific mutation in the KRAS codon 13, namely, p.G13D, has been associated with improved outcomes amongst patients with stage IV colorectal cancer treated with cetuximab both in the first-line and refractory settings, which has to be further validated in future studies [20, 21]. Thus, KRAS mutations have both predictive and prognostic value amongst patients with colorectal cancer. Furthermore, there are accumulating data indicating that BRAF mutations may also have a predictive value for patients with colon cancer. In the randomised Southwest Oncology Group (SWOG) 1406 trial, patients with BRAF ${ }^{V 600 E}$-mutant and all RAS wildtype metastatic colorectal cancer were treated with the combination of cetuximab and irinotecan, with or without vemurafenib, a BRAF inhibitor. Although no complete responses were reported, patients treated with vemurafenib had a significantly prolonged PFS compared with the others (median: 4.4 months vs. 2.0 months, $p<0.001$ ) [22]. Recently, the final results of the phase 3 BEACON clinical trial confirmed the value of BRAF mutational status. About 665 patients with the BRAF $^{V 600 E}$ mutation were randomised to receive either encorafenib, which is a BRAF inhibitor, binimetinib, which is a MEK inhibitor, and cetuximab (triplet-therapy group) or encorafenib and cetuximab (doublet-therapy group) or the investigators' choice including either cetuximab and irinotecan or cetuximab and FOLFIRI (folinic acid, fluorouracil and irinotecan) (control group). The study met its primary end point by demonstrating a significant OS benefit in the triplet-therapy group with a median OS of 9 months (vs. 5.4 months in the control group; $p<0.001)$. Patients receiving the doublet therapy also had an improved OS compared with the control group (median OS: 8.4 months vs. 5.4 months; $p<0.001$ ). The adverse event rates were similar amongst the three treatment groups [23].

Tailoring cancer therapeutics is the cornerstone of the current management of patients with cancer in order to provide the right drug combination to the right patient in the right setting. The expression of PD-L1 and the expression of KRAS in lung and colon carcinomas, respectively, are two representative cases amongst several biomarkers that have already been incorporated into the therapeutic algorithms. The emergence of minimally invasive liquid biopsies is also a promising approach and may provide a real-time overview of the evolving molecular landscape of the tumour, as reviewed in [24]. However, there is a huge number of potential biomarkers that have not shown significant clinical utility mainly because of lack of standardisation (different analytical techniques, different cut-off values amongst the assays, use of archival or fresh samples) and validation (sensitivity, specificity, positive and negative predictive value), as reviewed in [25, 26]. Therefore, a systemic evaluation of biomarkers is necessary in the early steps of drug development in order for the most suitable ones to be included in subsequent clinical trials as surrogate end points of response and survival.

\section{Disclosure of Conflicts of Interest}

All other authors report no relevant conflict of interest.

\section{References}

[1] Meric-Bernstam F, Mills GB. Overcoming implementation challenges of personalized cancer therapy. Nat Rev Clin Oncol 2012; 9: 542-548.

[2] Biomarkers Definitions Working G. Biomarkers and surrogate endpoints: preferred definitions and conceptual framework. Clin Pharmacol Ther 2001; 69: 89-95.

[3] Bray F, Ferlay J, Soerjomataram I et al. Global cancer statistics 2018: GLOBOCAN estimates of incidence and mortality worldwide for 36 cancers in
185 countries. CA Cancer J Clin 2018; 68: 394-424.

[4] Hirsch FR, Scagliotti GV, Mulshine JL et al. Lung cancer: current therapies and new targeted treatments. Lancet 2017; 389: 299-311.

[5] Zielinski C, Knapp S, Mascaux C et al. Rationale for targeting the immune system through checkpoint molecule blockade in the treatment of non-smallcell lung cancer. Ann Oncol 2013; 24: 1170-1179.

[6] Herbst RS, Baas P, Kim DW et al. Pembrolizumab versus docetaxel for previously treated, PD-L1- 
positive, advanced non-small-cell lung cancer (KEYNOTE-010): a randomised controlled trial. Lancet 2016; 387: 1540-1550.

[7] Herbst RS, Baas P, Perez-Gracia JL et al. Use of archival versus newly collected tumor samples for assessing PD-L1 expression and overall survival: an updated analysis of KEYNOTE-010 trial. Ann Oncol 2019; 30: 281-289.

[8] Reck M, Rodriguez-Abreu D, Robinson AG et al. Pembrolizumab versus Chemotherapy for PD-L1Positive Non-Small-Cell Lung Cancer. N Engl J Med 2016; 375: 1823-1833.

[9] Reck M, Rodriguez-Abreu D, Robinson AG et al. Updated Analysis of KEYNOTE-024: Pembrolizumab Versus Platinum-Based Chemotherapy for Advanced Non-Small-Cell Lung Cancer With PD-L1 Tumor Proportion Score of 50\% or Greater. J Clin Oncol 2019; 37: 537-546.

[10] Gandhi L, Rodriguez-Abreu D, Gadgeel S et al. Pembrolizumab plus Chemotherapy in Metastatic Non-Small-Cell Lung Cancer. N Engl J Med 2018; 378: 2078-2092.

[11] Ready N, Hellmann MD, Awad MM et al. FirstLine Nivolumab Plus Ipilimumab in Advanced Non-Small-Cell Lung Cancer (CheckMate 568): Outcomes by Programmed Death Ligand 1 and Tumor Mutational Burden as Biomarkers. J Clin Oncol 2019; JCO1801042.

[12] Ciardiello F, Tortora G. EGFR antagonists in cancer treatment. N Engl J Med 2008; 358: 1160-1174.

[13] Schmoll HJ, Van Cutsem E, Stein A et al. ESMO Consensus Guidelines for management of patients with colon and rectal cancer. a personalized approach to clinical decision making. Ann Oncol 2012; 23: 2479-2516.

[14] Di Fiore F, Blanchard F, Charbonnier F et al. Clinical relevance of KRAS mutation detection in metastatic colorectal cancer treated by Cetuximab plus chemotherapy. $\mathrm{Br} \mathrm{J}$ Cancer 2007; 96: 11661169.

[15] Lievre A, Bachet JB, Boige $V$ et al. KRAS mutations as an independent prognostic factor in patients with advanced colorectal cancer treated with cetuximab. J Clin Oncol 2008; 26: 374-379.

[16] Karapetis CS, Khambata-Ford S, Jonker DJ et al. K-ras mutations and benefit from cetuximab in advanced colorectal cancer. N Engl J Med 2008; 359: 1757-1765.

[17] Amado RG, Wolf M, Peeters M et al. Wild-type KRAS is required for panitumumab efficacy in patients with metastatic colorectal cancer. J Clin Oncol 2008; 26: 1626-1634.
[18] Bokemeyer C, Van Cutsem E, Rougier P et al. Addition of cetuximab to chemotherapy as first-line treatment for KRAS wild-type metastatic colorectal cancer: pooled analysis of the CRYSTAL and OPUS randomised clinical trials. Eur J Cancer 2012; 48: 1466-1475.

[19] Douillard JY, Siena S, Cassidy J et al. Randomized, phase III trial of panitumumab with infusional fluorouracil, leucovorin, and oxaliplatin (FOLFOX4) versus FOLFOX4 alone as first-line treatment in patients with previously untreated metastatic colorectal cancer: the PRIME study. J Clin Oncol 2010; 28: 4697-4705.

[20] De Roock W, Jonker DJ, Di Nicolantonio F et al. Association of KRAS p.G13D mutation with outcome in patients with chemotherapy-refractory metastatic colorectal cancer treated with cetuximab. JAMA 2010; 304: 1812-1820.

[21] Tejpar S, Celik I, Schlichting $M$ et al. Association of KRAS G13D tumor mutations with outcome in patients with metastatic colorectal cancer treated with first-line chemotherapy with or without cetuximab. J Clin Oncol 2012; 30: 3570-3577.

[22] Kopetz S, Mcdonough S, Lenz H-J et al. Randomized trial of irinotecan and cetuximab with or without vemurafenib in BRAF-mutant metastatic colorectal cancer (SWOG S1406) J Clin Oncol. 2017;35(15 Suppl):3505.

[23] Kopetz S, Grothey A, Yaeger R et al. Encorafenib, Binimetinib, and Cetuximab in BRAF V600EMutated Colorectal Cancer. N Engl J Med 2019; 381: 1632-1643.

[24] Rachiglio AM, Esposito Abate R, Sacco A et al. Limits and potential of targeted sequencing analysis of liquid biopsy in patients with lung and colon carcinoma. Oncotarget 2016; 7: 66595-66605.

[25] Butterfield LH. The Society for Immunotherapy of Cancer Biomarkers Task Force recommendations review. Semin Cancer Biol 2018; 52: 12-15.

[26] Manicone M, Poggiana C, Facchinetti A et al. Critical issues in the clinical application of liquid biopsy in non-small cell lung cancer. J Thorac Dis 2017; 9: S1346-S1358. 